\title{
An Integrated Metaheuristic Routing Method for Multiple-Block Warehouses with Ultranarrow Aisles and Access Restriction
}

\author{
Fangyu Chen $\mathbb{D}^{1},{ }^{1}$ Gangyan $\mathrm{Xu},{ }^{2}$ and Yongchang Wei ${ }^{1}{ }^{1}$ \\ ${ }^{1}$ Institute of Operations Management and System Engineering, School of Business Administration, \\ Zhongnan University of Economics and Law, Wuhan 430073, China \\ ${ }^{2}$ School of Architecture, Harbin Institute of Technology, Shenzhen, China
}

Correspondence should be addressed to Yongchang Wei; ivanwilts306@163.com

Received 4 April 2019; Accepted 26 May 2019; Published 18 June 2019

Guest Editor: Jorge Luis García-Alcaraz

Copyright (c) 2019 Fangyu Chen et al. This is an open access article distributed under the Creative Commons Attribution License, which permits unrestricted use, distribution, and reproduction in any medium, provided the original work is properly cited.

A problem-specific routing algorithm integrating ant colony optimization (ACO) and integer-coded genetic algorithm (GA) is developed to address the newly observed limitations imposed by ultranarrow aisles and access restriction, which exist in the largest e-commerce enterprise with self-run logistics in China. Those limitations prohibit pickers from walking through the whole aisle, and the access restriction even allows them to access the pick aisles only from specific entrances. The ant colony optimization is mainly responsible for generating the initial chromosomes for the genetic algorithm, which then searches the near-optimal solutions of picker-routing with our novel chromosome design by recording the detailed information of access modes and subaisles. To demonstrate the merits of the proposed algorithm, a comprehensive simulation for comparison is conducted with 12 warehouse layouts with real order information. The simulation results show that the proposed hybrid algorithm is superior to dedicated heuristics in terms of solution quality. The impacts of the parameters with respect to warehouse layout on the picking efficiency are analyzed as well. Setting more connect aisles and cross aisles is suggested to effectively optimize the picking-service efficiency in the presence of access limitations.

\section{Introduction}

For most e-commerce enterprises or online distributors, a well-designed order-picking strategy (from warehouse location [1] to routing method [2]) can promote the efficiency of the entire supply chain [3]. Given the advent of e-commerce, the significance of order-picking has grown $[4,5]$. Therefore, we concentrate on a picker-routing problem based on the observation in a Chinese e-commerce enterprise.

The order service time is one of the main performance measurements of warehouse systems. In a picker-to-parts warehouse, Petersen [6] pointed out that service time can be represented as a positive correlation function of one picker's travel distance. Accordingly, an appropriate routing method that identifies the shortest picking route can minimize the order service time and therefore improve the picking efficiency. One alternative option that is capable of reducing warehouse management costs is to increase the space utilization, which is typically made by warehouse owners to minimize the pick aisles' width, culminating in the widespread adoption of the narrow-parallel aisles in picker-to-parts warehouses. In a narrow-aisle warehouse with multiple order pickers, congestions may happen [7].

Recently, we observed an interesting warehouse system with ultranarrow aisles in a realistic Chinese online retailer with self-run logistics, $D$ Company, meaning that the pick aisles' widths are narrowed further, relative to the aforementioned narrow aisles. The ultranarrow aisles impede order pickers to enter while pushing their picking carts. They should deposit their carts at the entrance. When all the picks are finished, they should walk out the aisles to get their carts. The connect aisles and cross aisles, which are located in the warehouse, allow the pickers to change working aisle, because the width of them is sufficient for walking with carts, as shown 


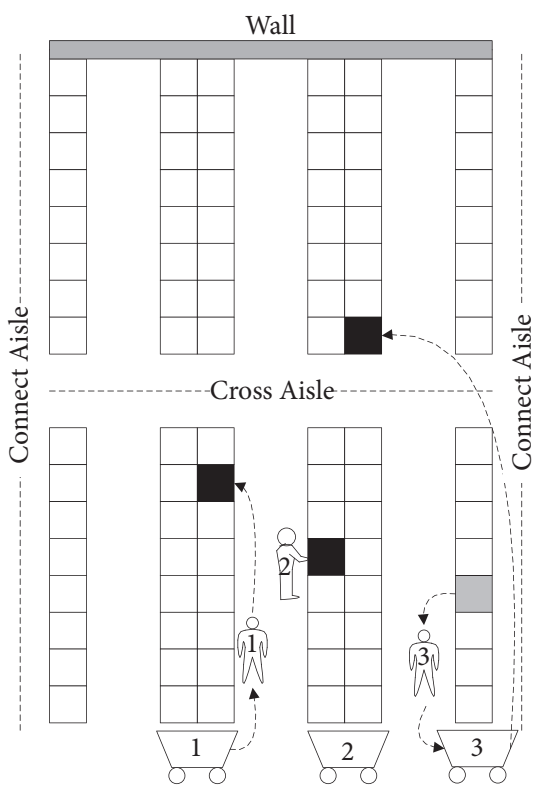

: Target Pick

: Finished Pick

FIGURE 1: Order picking in the ultranarrow aisles.

in Figure 1. For such a warehouse, there is little utility for existing routing methods that permit the order pickers to walk through the whole pick aisles. Additionally, constraints in physical space lead to access restrictions. For example, the racks that are located in the front of a warehouse system may be placed against the front wall; order pickers can only access these aisles through the back entrances. To promote the ecommerce boom, this paper aims to propose efficient routing methods for the warehouses taking both ultranarrow aisles and access restriction into consideration.

We propose a method by integrating ant colony optimization $(A C O)$ and genetic algorithm $(G A)$, which is named as AGNA (hybrid ACO and GA for ultranarrow aisles and access restriction). The novelties of AGNA are as follows. Firstly, to avoid traversing aisles, all the aisles with picks are restricted to be visited by specified access modes, and the access mode will map the picks at the certain entrances. Secondly, to deal with the unique characteristics induced by access restriction, the mechanism of determining the feasible travel routes between designated entrances is embedded in AGNA. Thirdly, to improve the convergence speed and performance of the routing method, $A C O$ is responsible for generating the initial chromosome for $G A$, and the integer-coded $G A$ is designed to search the near-optimal solutions mainly through our novel chromosome design that represents all the access modes for all the aisles with picks. To validate the effectiveness of the improved routing method, a comprehensive simulation study was performed with the data collected from the aforementioned $D$ company. Meanwhile, the impact of the warehouse layout is elucidated. We found that the AGNA method can be readily deployed in different warehouse-layout scenarios due to its flexibility.
The main contributions of this research lie in the following aspects. Firstly, we developed a hybrid AGNA-based routing method to address the methodological challenges for the warehouses with ultranarrow aisles and access restriction. To cope with the unique characteristics of routing problem in this kind of warehouses, many innovative mechanisms with respect to feasibility judgment and chromosome coding are significantly incorporated in our hybrid AGNA-based algorithm. Secondly, with the real data of customer order information and warehouse configuration collected from the $D$ company, a simulation study is employed to show the effectiveness and efficacies of the proposed method. The negative impacts brought by new features are analyzed as well.

The remainder of this paper is arranged as follows. Section 2 presents a review of the related literature and introduces some existing dedicated heuristics for singleblock warehouses. Some of these heuristics can be applied in the warehouse concerned in this paper after improvement, and the modified heuristics are compared with our designed AGNA method. In Section 3, the optimization model tailored to the concerned routing problem in the warehouse is defined. The AGNA is presented in Section 4. A comprehensive simulation is implemented to validate the efficiency of the proposed method in Section 5. Finally, the paper is concluded in Section 6 and our ongoing research topics are introduced as well.

\section{Literature Review}

The literature closely related to our research can be grouped into two main streams: one is the impact of aisles width, which constitutes a key feature in our research, while the other is the picker-routing problem. In the following, we will review these two streams of literature separately.

2.1. The Impact of Aisle Width. Reducing the pick aisles' width is an alternative to enlarge the space utilization [7]. Nevertheless, narrow aisles lead to more frequent congestion. In this regard, by changing the ratio of the pick: walktime, analytical models to assess the order picker congestion (blocking) in both narrow-aisle and wide-aisle warehouses are developed by Parikh and Meller [8, 9]. Hong et al. [10] proposed a batching method in warehouses with narrow aisle. Similarly, Chen et al. [11, 12] introduced some routing methods for narrow-aisle warehouses with the consideration of congestion.

Most of the previous literature primarily considers the traditional wide-aisle or narrow-aisle warehouse systems. Although Chen et al. [13] proposed a set of heuristic routing methods for the ultranarrow aisle warehouse system with consideration of access restriction, these heuristics are developed on the existing methods for basic warehouse layout. These methods have the advantage in the ease of implementation. Nevertheless, they can deviate from the optimal solutions seriously, which further increase operational costs. To this end, to get a better optimization effect on picking efficiency, we concentrate on designing a judicious routing method based on metaheuristics. 
2.2. Routing Method. We can divide all the existing routing methods in the related literature into two groups: dedicated heuristics and metaheuristics. The dedicated heuristics have found prevalent application for order picking, given the ease of their deployment [14-16]. In the existing dedicated heuristics, only the Largest Gap and Return methods forbid the order pickers from traversing the subaisles for most situations, as shown in Figure 2. However, these two methods cannot be readily deployed in the warehouse system concerned in this paper. For example, the Largest Gap method requests the order pickers to walk through the first aisle with picks to reach the back of the block.

For more complicated environments, metaheuristics are more suitable [17-19]. Except for generating picking routes in complicated environments such as multiple-order pickers [12], much of the relevant literature has focused on designing integrated solutions for both order-batching and pickerrouting [20-22]. Although many metaheuristics have been presented, such as Particle Swarm Optimization [23] and Simulated Annealing [24], ACO is the most popular for the picker-routing problem [25-27]. To design the picking route for the warehouse of interest in our research, we design a routing method by integrating $A C O$ and $G A$. This integrated method can dynamically control the invocation time of both $A C O$ and GA and improve the convergence speed and performance of the algorithm.

\section{Problem Definition}

3.1. Warehouse Configuration and Assumption. The related literature $[4,28]$ and practice focus commonly on a multipleblock picker-to-parts warehouse, which is concerned in our paper as well, as shown in Figure 3. The main difference of our research is that the widths of all the parallel pick aisles are assumed to be ultranarrow. Additionally, some aisles may have access restriction on designated entrances. The $C A s$ (cross aisles) are located in the front-back direction. Likewise, there are CNA (connect aisles) in the left-right direction. The widths of $C A$ and $C N A$ are sufficient to permit order pickers walking with their picking carts, which allows order pickers to change working aisles. The cross point is the intersection between a $C A$ and a $C N A$. The warehouse is divided into multiple blocks $(B)$ by these $C A s$ and $C N A$ s in front-back and left-right directions. Each single pick aisle is also divided into a number of subaisles $(S)$.

A Row contains the blocks arranged in the left-right direction, and correspondingly a Column refers to those in the front-back direction. For an $S, E_{f}$ and $E_{b}$ indicate the front entrance and back entrance, respectively. For a single block, there are two adjacent $C A s$, one is $C A_{f}$ (front cross aisle), and the other is $C A_{b}$ (back cross aisle). For an order picker, the working cross aisle is denoted as $C A_{c}$ (current cross aisle). To describe a route from one subaisle to another, $S_{b}$ (Begin Subaisle) denotes the subaisle where the order picker begins, and $S_{t}$ (Target Subaisle) denotes the subaisle which the order picker is going to visit. Moreover, the front entrances of $S_{b}$ and $S_{t}$ are $E_{b f}$ and $E_{t f}$, respectively, and their back entrances are $E_{b b}$ and $E_{t b}$, respectively. Given this layout, some assumptions are noteworthy. Firstly, in such a warehouse, the two-sided picking policy is chosen by the order pickers [29]. Meanwhile, we ignore the time cost on reaching the different layers and sides of shelves.

In the warehouse of e-commerce enterprise, the dynamic arrival of customers' orders means that we can composite all the orders into batches depending only on their arrival time. We assume that the warehouse adopts random-storage policy [30]. We impose restriction on the capacity of picking carts to avoid overload [31], and the picker congestion is ignored. For simplicity, we ignore the difference in the order pickers' walking speed and the time of finishing a picking task is assumed to be constant $[32,33]$. The order pickers have no constraint and can change walking direction in any aisles [34]. The picking operation begins and finishes at the depot, where the operating time is ignored in this paper.

\subsection{Routing Model. According to the Largest Gap and Return} methods, to avoid traversing and deal with the access restriction, the most effective picking solution for each subaisle with picks can be illustrated by three access modes: Frontreturn, Back-return, and Gap-return (as shown in Figure 4). The Front-return mode inquires the order pickers to walk into and depart from a subaisle through the front entrance after visiting all the picks. Correspondingly, in the Backreturn mode, the order pickers only use the back entrance for both entry and departure. In the Gap-return mode, the order pickers enter a pick aisle but make a U-turn at the largest gap. The order pickers may have to visit the subaisle twice: once by $E_{f}$ and another by $E_{b}$. Because the largest gap may cut the subaisle into two parts, this implies that the Gap-return mode corresponds to two access modes: accessing the front part and the back part. The Gap-return mode does not necessitate the order pickers to visit the remaining part immediately after they finish one part of a subaisle. Once the access mode is determined for a subaisle, the picking route in this subaisle is determined as well, and the picks therein will be mapped to the entrance which should be visited by the order pickers. The Front-return mode will map all the picks to $E_{f}$, the Back-return mode will map all the picks to $E_{b}$, and the Gap-return mode will map the picks to $E_{f}$ and $E_{b}$, respectively. Then, routing methods only need to find a satisfactory moving route passing all the entrances with mapped picks. Finally, an integrated route is generated by combining this moving route and the picking routes in subaisles with picks. Consequently, the routing problem can be equivalently expressed by determining the best access modes and finding the best visiting sequence for all the subaisles with picks.

Based on the assumptions and the access modes aforementioned, the objective is to minimize the total travel distance of serving a batch. Then, we can formulate a $0-1$ integer linear optimization model.

Indices and Sets

$\Omega$ : Set of subaisles with picks and the depot

$i, j \in \boldsymbol{\Omega}$ : Indexes of subaisles with picks and depot, in which $i=0$ means the depot 


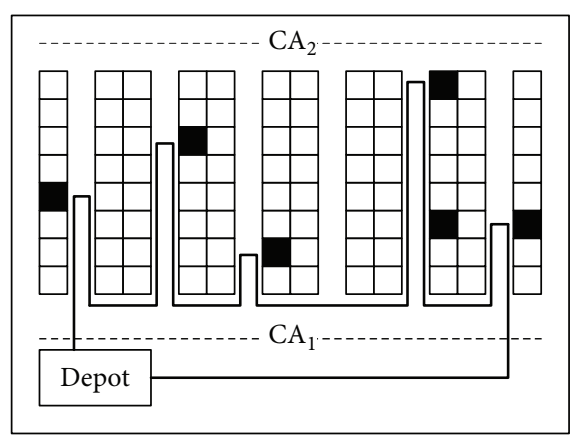

(a) Return from single block

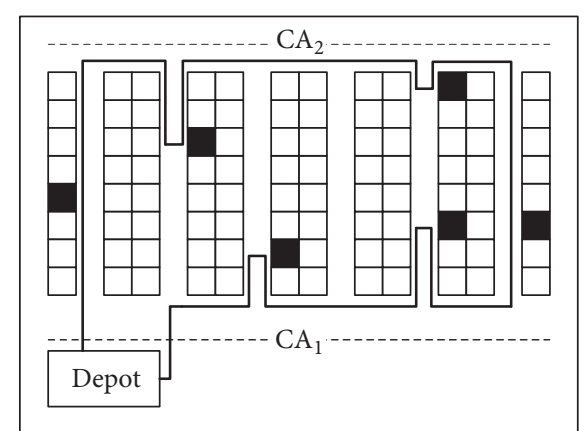

(b) Largest Gap from single block

FIgURE 2: A sketch of Return and Largest Gap.

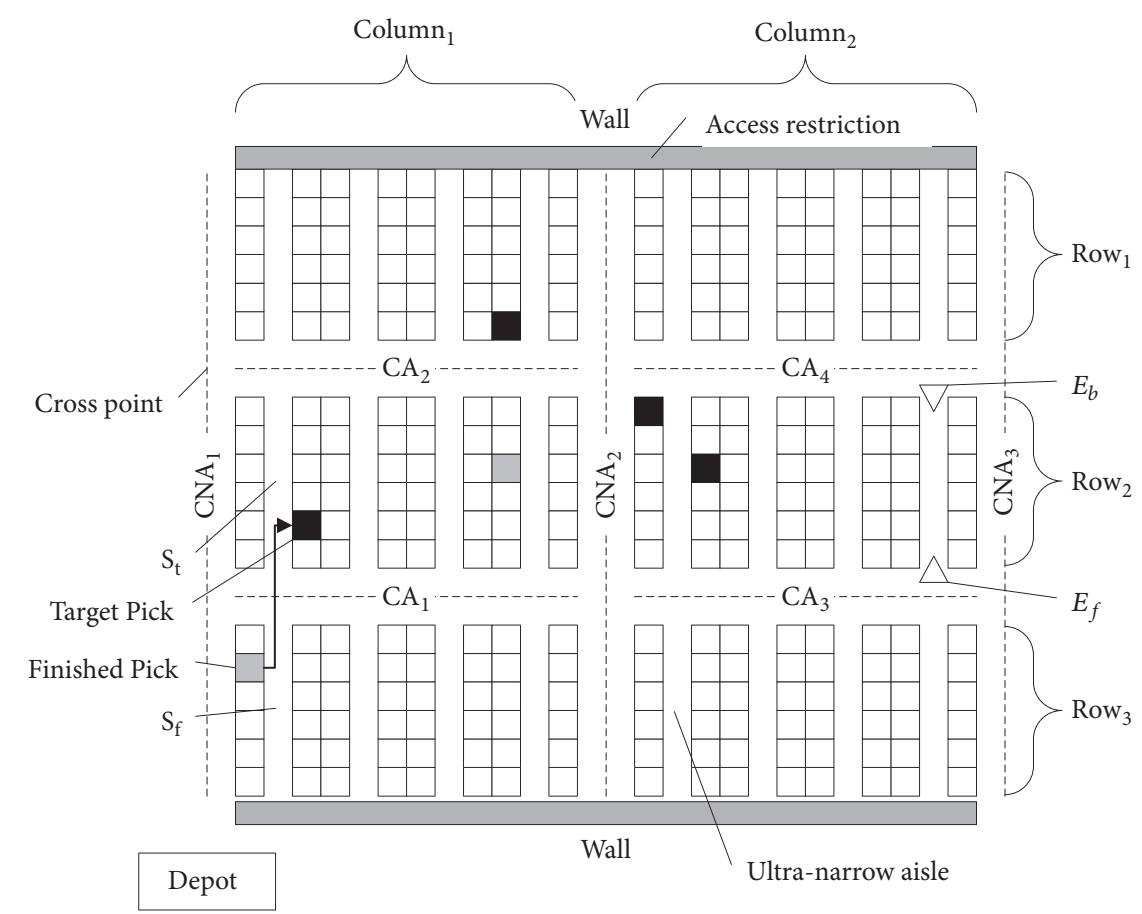

FIGURE 3: Multiple-block warehouse system with ultranarrow aisles and access restriction.

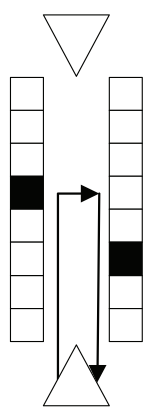

Front-return

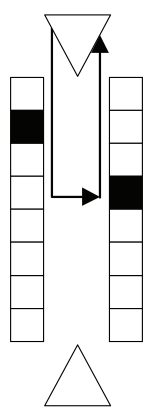

Back-return

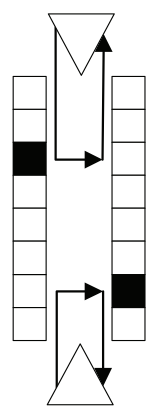

Gap-return

FIgURE 4: Access modes for subaisles with picks.

$\mathbf{R}_{i, j}$ : Set of the feasible moving routes from subaisles $i$ to $j$

$r \in \mathbf{R}_{i, j}$ : Index of the feasible moving routes from subaisles $i$ to $j$

$\mathbf{M}_{j}$ : Set of the feasible access modes of subaisle $j$

$m \in \mathbf{M}_{j}$ : Index of the feasible access modes of subaisle j

\section{Parameters and Sets}

$\mathbf{D}_{i j}$ : Set of the moving distances of the feasible moving routes from subaisles $i$ to $j$

$d_{i j r} \in \mathbf{D}_{i j}$ : Moving distance of the $r^{\text {th }}$ feasible moving route from subaisles $i$ to $j$

$\mathbf{D}^{\prime}{ }_{j}$ : Set of the picking distances of the feasible access modes of subaisle $j$ 
$d_{j m}^{\prime} \in \mathbf{D}_{j}^{\prime}$ : Picking distance of the $m^{\text {th }}$ feasible access mode of subaisle $j$

\section{Decision Variables}

$x_{i j}$ : Binary decision that equals $1\left(x_{i j}=1\right)$ means that subaisle $i$ is visited directly after subaisle $j$

$y_{i j r}$ : Binary decision that equals $1\left(y_{i j r}=1\right)$ means that the $r^{\text {th }}$ feasible moving route is selected to move from subaisles $i$ to $j$

$z_{j m}$ : Binary decision that equals $1\left(z_{j m}=1\right)$ means that the $m^{\text {th }}$ feasible access mode is selected to visit subaisle $i$ to $j$

\section{Model Formulation}

$$
\min \left\{\sum_{i \in \boldsymbol{\Omega}} \sum_{j \in \boldsymbol{\Omega}} x_{i j}\left(\sum_{k \in \mathbf{T}_{i j}} y_{i j r} d_{i j r}+\sum_{m \in \mathbf{M}_{j}} z_{j m} d^{\prime}{ }_{j m}\right)\right\}
$$

subject to

$$
\begin{aligned}
\sum_{r \in \mathbf{R}_{i j}} y_{i j r} & =1, \quad \forall i, j \in \mathbf{\Omega} \\
\sum_{m \in \mathbf{M}_{j}} z_{j m} & =1, \quad \forall j \in \mathbf{\Omega} \\
x_{i j} & \in\{0,1\}, \quad \forall i, j \in \mathbf{\Omega} \\
y_{i j r} & \in\{0,1\}, \quad \forall i, j \in \mathbf{\Omega}, \forall r \in \mathbf{R}_{i j} \\
z_{j m} & \in\{0,1\}, \quad \forall j \neq 0 \in \mathbf{\Omega}, \forall m \in \mathbf{M}_{j}
\end{aligned}
$$

Function (1) is the objective function that minimizes the total travel distance of serving a batch. Constraint (2) ensures that only one moving route is selected when the order pickers move from subaisle $i$ to $j$. Constraint (3) ensures that each subaisle selects only one access mode. Constraints (4) to (6) define the variable domains.

The routing model differs from the basic picker-routing problem, which can be defined as the Steiner-TSP (Steiner Travelling Salesman Problem) [14]. All the cities (subaisles) in the TSP need to be visited only once. However, if a subaisle (city) chooses the Gap-return mode, the order pickers may visit this subaisle twice: once by $E_{f}$ and another time by $E_{b}$. For our model, this removes the necessity for the common constraint in TSP that each subaisle (city) has exact only one predecessor and successor. Besides, when the Gap-return mode is determined for a subaisle and the order pickers are required to visit $E_{f}$ immediately after $E_{b}$, the common constraint in TSP that there is only a single tour is likewise rendered unnecessary for our model.

Furthermore, the impact of access restriction cannot be incorporated into the model. When the selected blocks have access restriction, some access modes will become infeasible for the subaisles. For example, if $C A_{f}$ of a block has access restriction, only the Back-return mode may be chosen for the subaisles in this block. Moreover, the choice of the access mode will affect the moving route between subaisles. For example, if the Front-return mode is selected for both $S_{b}$ and $S_{t}$, the order pickers will have to enter and leave these subaisles by $E_{b f}$ and $E_{t f}$, signifying that the moving route between $S_{b}$ and $S_{t}$ must pass $E_{b f}$ and $E_{t f}$.

Generally, there is an interaction between the values of $Z_{j m}$ and $y_{i j r}$. Before the routing-construction begins, for each subaisle, we can just get a set of the "selectable" access modes and the corresponding detailed picking routes for these access modes are also contingent upon the access restriction constraint. The set of the "selectable" moving routes between any two subaisles can be confirmed in this stage as well.

When the order picker chooses the access mode for $S_{t}$, by extracting from the set of the "selectable" moving routes, we can determine the set of feasible moving routes between $S_{b}$ and $S_{t}$. The access mode of the current $S_{t}$ will also affect the choice of the moving route from the current $S_{t}$ to the next $S_{t}$, once the access mode of the next $S_{t}$ is determined. Due to the aforementioned characteristics, the accurate set of $\mathbf{D}_{i j}$ can not be determined prior to the selection of optimal routing solutions. Thus, our problem cannot be solved simply by optimization solvers, such as Lingo and CPLEX, which motivates us to design metaheuristic-based routing methods.

\section{Solution Methods}

In this section, a hybrid algorithm integrating $A C O$ (ant colony optimization) and integer-coded $G A$ (genetic algorithm) is developed to deal with the specified routing problem. On one hand, the ACO generates the initial chromosome for the integer-coded $G A$ during the initialization. This structure can not only dynamically control the invocation time of both the GA and ACO but also improve the convergence speed and performance of the algorithm. On the other hand, the integer-coded $G A$ searches a near-optimal solution for picker-routing. The total travel distance of serving a batch could be defined as the fitness values of the integer-coded chromosomes obtained from the $G A$.

4.1. Distance Initialization. For different access modes of one subaisle, the corresponding picking distances and picking routes differ from each other. Similarly, the moving route and moving distance between $S_{b}$ and $S_{t}$ are also affected by their access modes. The access mode for a subaisle can be determined only when this subaisle is chosen as $S_{t}$. After that, its picking route and moving route can be correspondingly confirmed. To represent the routing problem as a combination optimization problem manageable by the AGNA, all the picking routes in subaisles and the moving routes between any two subaisles for different access modes should be recorded during the initialization.

For a subaisle, each access mode will determine the corresponding picking route and picking distance. This applies especially to the Gap-return mode, in which the front and the back parts lead to different picking routes and picking distances. This means that, for each subaisle without access restriction, four kinds of picking routes are usually available: for the Front-return mode, for the Back-return mode, for the 
front part of the Gap-return mode, and for the back part of the Gap-return mode. In the initialization, all the corresponding picking routes and picking distances will be calculated and recorded as the input for the routing method.

Moreover, there are four cases of the moving route and moving distance between $S_{b}$ and $S_{t}$, which are affected by the access modes of $S_{b}$ and $S_{t}$.

Case 1. The Front-return mode is chosen for both $S_{b}$ and $S_{t}$, or the Gap-return mode is chosen for both of them, in which only the front part of $S_{b}$ is completed and the front part of $S_{t}$ needs to be visited. Then, the moving route will start from $E_{b f}$ and end at $E_{t f}$.

Case 2. The Front-return mode is chosen for $S_{b}$, or the Gap-return mode is chosen, in which only the front part is completed. Conversely, the Back-return mode is chosen for $S_{t}$, or the Gap-return mode is chosen, in which only the back part needs to be visited. Then, the moving route will start from $E_{b f}$ and end at $E_{t b}$.

Case 3. The Back-return mode is chosen for $S_{b}$, or the Gapreturn mode is chosen, in which only the back part is completed. Conversely, the Front-return mode is chosen for $S_{t}$, or the Gap-return mode is chosen, in which only the front part needs to be visited. Then, the moving route will start from $E_{b b}$ and end at $E_{t f}$.

Case 4. The Back-return mode is chosen for both $S_{b}$ and $S_{t}$, or the Gap-return mode is chosen for both of them, in which only the back part of $S_{b}$ is completed and the back part of $S_{t}$ needs to be visited. Then, the moving route will start from $E_{b b}$ and end at $E_{t b}$.

It deserves attention that the moving route and its moving distance between $S_{b}$ and $S_{t}$ still need to be computed for each case. The moving distance is usually calculated by the path between any two designated entrances with the Manhattan distance (Theys et al., 2010). However, the Manhattan distance cannot fully satisfy the layout with the ultranarrow aisles. According to the diversified spatial relationship between the designated entrances of $S_{b}$ and $S_{t}$, as shown in Figure 5, the detailed definitions of the moving route and moving distance are described as follows [13].

(i) Entrances in the Same CA. The order pickers can walk directly from the designated entrance of $S_{b}$ to the designated entrance of $S_{t}$; see Route (a) shown in Figure 5.

(ii) Entrances in Different CAs but in the Same Column. Usually, two optional moving routes between the designated entrance of $S_{b}$ and the designated entrance of $S_{t}$ are available to the order pickers. For simplicity, the shorter one is chosen; refer to Route (b) shown in Figure 5.

(iii) Entrances in Different Columns. The order pickers walk along $C A_{c}$ by leaving $S_{b}$ from the chosen entrance, until getting to the CNA adjacent to the $C A$ which the chosen entrance of $S_{t}$ belongs to. Then, after setting this $C A$ as $C A_{c}$,

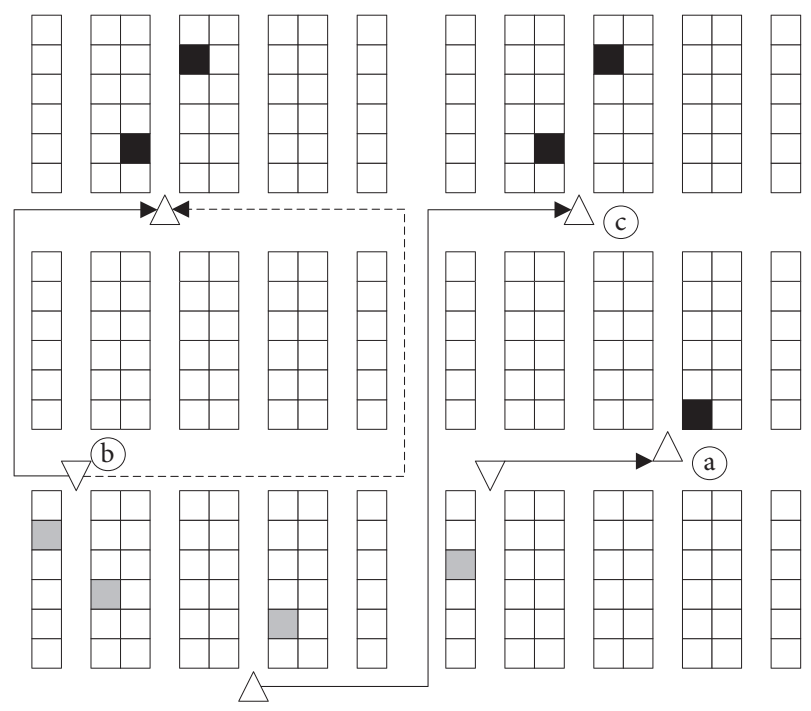

FIGURE 5: The definition rules of the moving routes.

the order pickers walk along $C A_{c}$ and enter $S_{t}$; see Route (C) shown in Figure 5.

All the moving routes and picking routes with respect to different access modes and their distances are recorded and will be used for route construction and fitness-value evaluation. If some access modes are infeasible due to access restriction, the related picking distance and moving distance will be recorded as infinity.

4.2. ACO-Based Chromosome Initialization. Based on the initialized distances, the $A C O$ is employed to initialize the population of the routing chromosomes. We assume that the ant colony contains $N(N \geq 2)$ ants, which denote the order pickers. The ant finds $S_{t}$ from the unfinished subaisles and determines the access mode of $S_{t}$. Next, it records the moving distance from $S_{b}$ to $S_{t}$ and the picking distance in $S_{t}$. When all the subaisles are finished, the ant will walk back to the depot. Then, $L$, the total travel distance, is composed by all the selected picking distances and moving distances, which will be seemed as the evaluation criterion. Before the $A C O$ starts, the initial value of the pheromone is calculated by

$$
\tau_{i j r}(0)=\frac{1}{\sum_{i \in \boldsymbol{\Omega}} \sum_{j \in \boldsymbol{\Omega}}\left|\mathbf{D}_{i j}\right|}
$$

where $\tau_{i j r}(0)$ is the pheromone concentration in the $r^{\text {th }}$ feasible moving route from subaisle $i$ to $j$ at time 0 and $\sum_{i \in \Omega} \sum_{j \in \Omega}\left|\mathbf{D}_{i j}\right|$ is the sum of the number of feasible moving routes among all the subaisles to be visited by the ants or order pickers.

Each ant chooses $S_{t}$ and determines the moving route randomly depending on the transition probability $p_{i j r}(t)$ that 
subaisle $j$ will be visited immediately after finishing subaisle $i$ at time $t$ by the $r^{\text {th }}$ moving route, which is represented as

$$
p_{i j r}(t)= \begin{cases}\frac{\left[\tau_{i j r}(t)\right]^{\alpha}\left[\eta_{i j r}\right]^{\beta}}{\sum_{j \in \mathbf{U}}\left[\tau_{i j r}(t)\right]^{\alpha}\left[\eta_{i j r}\right]^{\beta}}, & \text { if } j \in \mathbf{U}, \\ 0, & \text { otherwise, }\end{cases}
$$

where $\tau_{i j r}(t)$ is the pheromone concentration on the $r^{\text {th }}$ moving route from subaisle $i$ to $j$ at time $t, \eta_{i j r}$ measures the visibility on this route, and $\mathbf{U}$ is the set of unfinished subaisles. The value of $\eta_{i j r}$ is usually defined as the reciprocal of the sum of the moving distance of the $r^{\text {th }}$ moving route from subaisle $i$ to $j$ and the corresponding picking distances in subaisles $i$ and $j$. The definition of the transition probability leads to high superiority to the shortest travel and picking route, and $\tau_{i j r}(t)$ impels the familiar moving route and picking route is chosen by these ants. It is well recognized that the parameters $\alpha$ and $\beta$ coordinate the relative importance between the pheromone concentration and the route visibility $(\alpha \geq 1, \beta \geq 1)$. Usually, $\alpha$ and $\beta$ are valued according to the former observation.

When all the ants complete their picking tasks, the pheromone concentration on all the routes is updated by

$$
\tau_{i j r}(t+1)=(1-\rho) \tau_{i j r}(t)+\Delta \tau_{i j r}(t, t+1)
$$

where $\rho$ is the recession parameter of the pheromone concentration $(0<\rho<1) . \Delta \tau_{i j r}(t, t+1)$ is the alteration of the pheromone concentration laid on moving route $r$ from subaisle $i$ to subaisle $j$ between times $t$ and $t+1$, as determined by

$$
\Delta \tau_{i j r}(t, t+1)=\sum_{n=1}^{N} \Delta \tau_{i j r}^{n}(t, t+1)
$$

where $\Delta \tau_{i j r}^{n}(t, t+1)$ is the pheromone concentration left on the route $r$ from subaisles $i$ to $j$ by the $n^{\text {th }}$ ant from times $t$ to $t+1$, which is defined by

$$
\begin{aligned}
& \Delta \tau_{i j r}^{n}(t, t+1) \\
& \quad= \begin{cases}\frac{1}{L_{n}}, & \text { if the } n^{\text {th }} \text { ant chooses the } r^{\text {th }} \text { route to move from sub-aisle } i \text { to sub-aisle } j \text { between times } t \text { and } t+1, \\
0, & \text { otherwise, }\end{cases}
\end{aligned}
$$

where $L_{n}$ means the total travel distance of the picking route completed by the $n^{\text {th }}$ ant.

When all the ants complete the trips, some solutions will be saved in a queue $(\mathbf{Q})$. If a new solution outside the queue but better than the worst solution in the queue is found, it will replace that worst solution in the queue. The $A C O$ will stop after the iterations equal the predetermined number. When it terminates, the solutions saved in the queue will be used in the initialization of chromosomes in the integer-coded GA.

\subsection{Integer-Coded Genetic Algorithm (GA)}

4.3.1. Chromosomal Representation and Initial-Population Generation. During the route construction process by ACO, the order pickers choose $S_{t}$ first and then decide the access mode. The unselected access modes of $S_{t}$ may be ignored easily in the later iterations. To avoid converging to local optimum, the integer-coded GA generates the near-optimal solutions by searching the access modes and the visiting sequence of all the subaisles with picks. The value of each gene in a chromosome not only records the visiting sequence of a certain subaisle but also determines the access mode of this subaisle. In our algorithm, if a gene is associated with the $i^{\text {th }}$ subaisle and the $m^{\text {th }}$ mode, the value of this gene is defined as $2^{i-1} * 3^{m-1}$. As is corresponding to all the access modes, we set $m=1$ as the Front-return mode, $m=2$ as the Back-return mode, $m=3$ as accessing the front part by the Gap-return mode, and $m=4$ as accessing the front part by the Gap-return mode. Consequently, this value formula helps us identify the unique subaisle and access mode for each gene.

It is noteworthy that although the $A C O$ has already generated a visiting sequence passing all the subaisles with feasible access modes, this solution may be insufficiently effective and ignores other unselected access modes. The chromosomal representation should include all the subaisles and all the access modes for them, and the length of chromosome will be $\sum_{i \in \Omega}\left|\mathbf{M}_{i}\right|$. Therefore, the solution of $A C O$ can be used as the encoding basis for the first few genes, and the remaining genes of the chromosome will be supplemented by random encoding. Figure 6 illustrates an example of the integercoded chromosomal representation including four subaisles without access restriction, which means that the length of the chromosome is 16 . The solutions generated by $A C O$ are sequentially represented as $\{i=1, m=4\},\{i=4, m=1\}$, $\{i=2, m=3\},\{i=3, m=2\},\{i=2, m=4\}$, and $\{i=1, m=3\}$. Such sequential representation implies visits to all the subaisles with picks by the following sequence: (1) accessing the back part of the $1^{\text {st }}$ subaisle by the Gapreturn mode; (2) accessing the $4^{\text {th }}$ subaisle by the Front-return mode; (3) accessing the front part of the $2^{\text {nd }}$ subaisle by the Gap-return mode; (4) accessing the $3^{\text {rd }}$ subaisle by the Backreturn mode; (5) accessing the back part of the $2^{\text {nd }}$ subaisle by the Gap-return mode; and (6) accessing the front part of the $1^{\text {st }}$ subaisle by the Gap-return mode. Hence, in the chromosomal initialization, the first six genes will be valued as $27,8,18,12,54$, and 9 , whereas the remaining 10 genes will 


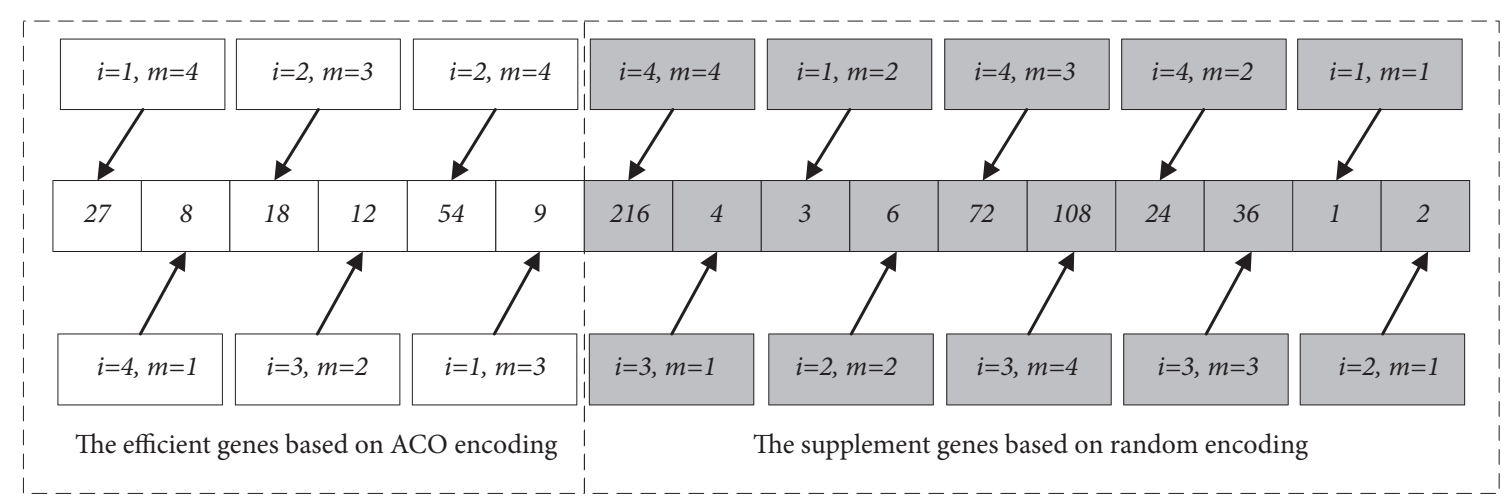

FIgURE 6: The illustration of integer-coding.

be randomly coded to cover the unselected access modes for further optimization.

The population of chromosome is $P$. When the encoding is finished for all the chromosomes, the crossover and mutation operation will begin.

4.3.2. Fitness Value Evaluation. The total travel distances of all the integer-coded chromosomes are chosen as their fitness values. Because each chromosome contains all the access modes for each subaisle with picks, not all the genes need to be calculated in the evaluation of the fitness values. For example, if the $i^{\text {th }}$ subaisle has already been indicated by a former gene to choose the Front-return mode, the remaining genes that indicate the $i^{\text {th }}$ subaisle to be accessed by other modes will be excluded. During the chromosomal decoding, the value of each gene should be validated to avoid any repeated or missed visits to the subaisles. If the subaisle is unfinished and the access mode is feasible, the gene will pass the validation and be calculated in the fitness values. The procedure of validation for each gene can be described as in Figure 7.

The calculation of the total travel distance of each chromosome is described as follows.

Step 1. Set $L=0$ and $S_{b}=S_{0}$ to signify that the order pickers start from the depot.

Step 2. Get the next gene.

Step 3. Decode the gene to get the subaisle and the access mode.

Step 4. If the subaisle $i$ and access mode $m$ pass the validation, set $S_{t}=S_{i}$; then proceed to Step 5; otherwise, go back to Step 2.

Step 5. Based on the access modes of $S_{b}$ and $S_{t}$, determine the moving route $r$ from $S_{b}$ to $S_{t}$. Get the moving distance $d_{b t r}$ and the picking distance $d_{t r}^{\prime}$; then set $L=L+d_{b t r}+d_{t r}^{\prime}$ and $S_{b}=S_{t}$.

Step 6. If all the subaisles are finished, go on with Step 7; otherwise, revert to Step 2.
Step 7. Return to the depot and determine the moving route $r$; set $S_{t}=S_{0}$ and $L=L+d_{b t r}$.

Step 8. The reciprocal value of $L$ is the fitness value of this solution.

Step 9. If $L$ is associated with a better fitness value than that of the near-optimal solution so far in the last population generation, set $L_{\text {near-optimal }}=L$.

4.3.3. Crossover and Mutation. The designating parents for the undergoing crossover and mutation are adopted by the approach of proportional selection. In this approach, the parents are selected randomly. However, the parents with higher fitness values tend to be selected with higher probability. Because the genes in the front of a chromosome are more likely to be calculated in the fitness value, the effective genes should be included in the crossover and mutation. To improve the evolutionary speed, the Four-Point Crossover method is adopted to create new offspring. Four segmentations with equal lengths are determined; the second and fourth gene segmentations are selected in crossover operation. Then, we use the Order Crossover in the Legality Repair. Figure 8(a) illustrates the crossover operation.

The mutation operation guarantees the diversity and avoids premature convergence. SBM (Swap-based mutation) - which selects two random elements (one from the first third part of the chromosome and the other from the remaining part) and exchanges their positions - is applied to generate the offspring. Figure 8(b) illustrates these mutation operators.

4.4. Stopping Criteria. The $G A$ algorithm terminates in two situations. The first one is as follows: once the iterations reaches a predetermined number of generations $G$, the $G A$ will terminate. The second criteria is that the $G A$ will terminate if the best solution remains the same for a fixed number of generations ep. The same criteria can be seen in the $A C O$ part, and the corresponding parameters are $I$ and $C$, respectively. Figure 9 describes the overall procedure of AGNA. 


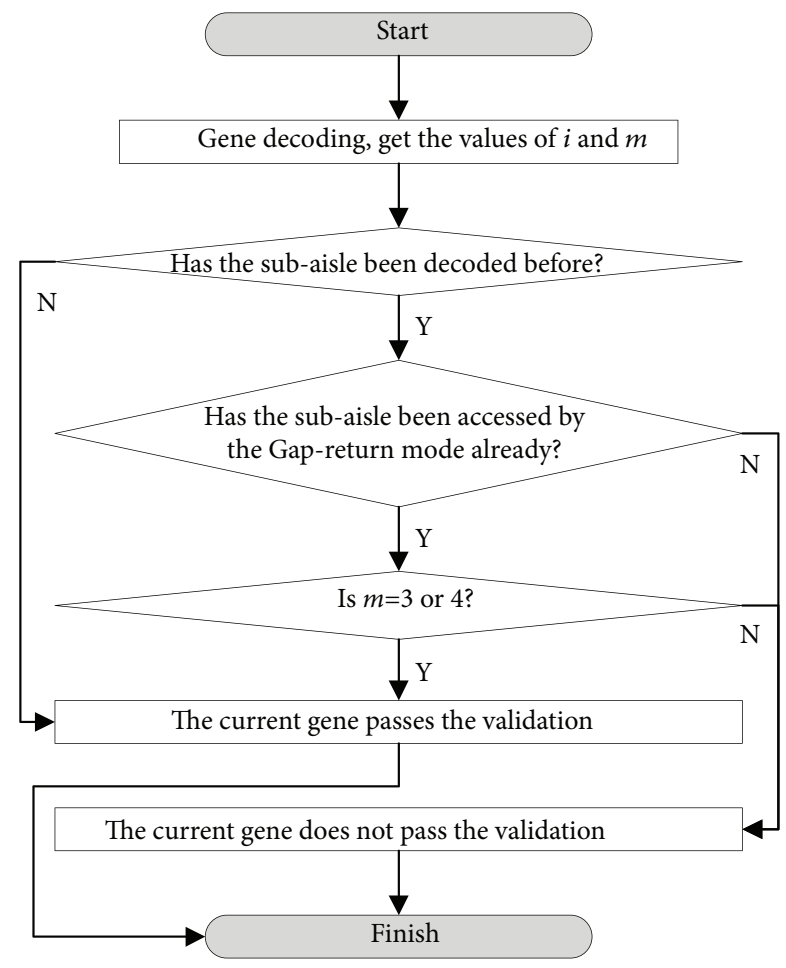

FIGURE 7: The procedure of validation for each single gene.

\section{Experimental Study}

5.1. Experimental Design. We conduct a comprehensive experiment to evaluate the effectiveness of the proposed method, which contains 12 kinds of warehouse layout scenarios extracted from a realistic warehouse system in $D$ company, as shown in Figure 10. The detailed configuration parameters are shown in Table 1, which is referred to Chen et al. [13].

There are usually at least 11 aisles in every single block. For each single subaisle, the minimum number of storage locations is 384 . The average length and width of each single subaisle are $12.048 \mathrm{~m}$ and $0.8 \mathrm{~m}$, respectively. For each single shelf, the average width is $0.7 \mathrm{~m}$. Furthermore, the average widths of each single $C N A$ and $C A$ are $3.64 \mathrm{~m}$ and $3.3 \mathrm{~m}$, respectively.

5.2. Experimental Results. The simulation is input with the realistic order information over one month in this aforementioned warehouse. For all the scenarios, at least 547,754 orders arrived in this month. The order information only contains the designated storage locations, without other customerrelated information. According to the practical situation, we restrict each picking cart to carry 60 items at most. Besides, there are usually only 1 to 2 items in an online customer order. To maximize the utilization of picking cart, orders are combined into batches. In this online warehouse, we use the Variable Time-Window Batching rule, also known as FirstCome-First-Served, to composite all the orders into batches according to their arrival time. After the batching operation, 13,060 batches are generated. There are 1,165 picking tasks at least assigned to the scenario with single block. To compare our hybrid AGNA method with the dedicated heuristics, we also improved the basic Return and Largest Gap methods to adapt to the warehouse concerned in this paper, which, respectively, are named as the RNA (Return for ultranarrow aisles and access restriction) and LNA (Largest Gap for ultranarrow aisles and access restriction). For the $R N A$ and $L N A$, each subaisle with picks also needs to choose access mode. The RNA has the Front-return and Back-return modes, and LNA has the Front-return, Back-return, and Gap-return modes. The procedures of RNA and LNA are described as follows [13].

Step 1. Sort and index all the CAs progressively in the Columns and then in the Rows.

Step 2. Choose feasible access modes with the shortest picking distance for all the subaisles with picks. Then, the picks will be mapped to the designated entrances.

Step 3. If the picking tasks are mapped to an entrance, the $C A$ with this entrance can be labelled as a $C A$ with picks.

Step 4. By traversing the indexes of the CAs incrementally, set the first found $C A$ with picks as $C A_{c}$; then the order pickers will walk to the left cross point of $C A_{c}$.

Step 5. Enter $C A_{c}$, and visit all the designated entrances with mapped picking tasks.

Step 6. If all the CAs with picks are visited, go on with Step 9; otherwise, go on with Step 7.

Step 7. If the next $C A$ with picks is in the same Column as $C A_{c}$, leave $C A_{c}$ from the nearer cross point; if the next $C A$ with picks is in different Columns, leave $C A_{c}$ from the right cross point of $C A_{c}$.

Step 8. Reach the next $C A$ with picks, and set this $C A$ as $C A_{c}$. Then, revert to Step 5 .

Step 9. Leave $C A_{c}$ and return to the depot.

The simulation platform is developed by C\#.net on a $4.00 \mathrm{GHz}$ PC. In all the AGNA solutions, the values of the following search parameters, as shown in Table 2, are validated to be robust in the previous research [11] and ANOVA test. For RNA and LNA, the average computation time for a batch was less than $1 \mathrm{~s}$. For $A G N A$, this time was less than $5 \mathrm{~s}$. The results show that, in eight scenarios, $A G N A$ gets the best performance. In the remaining four scenarios, the three routing methods get the same results, as shown in Table 3. Data in italic font means the best result in the corresponding scenario. Owing to the flexible definition of the visiting sequence for a subaisle, AGNA can optimize the travel route effectively. Additionally, the access modes are derived from existing basic heuristics, with which the order pickers are familiar. Accordingly, the order pickers can easily accept the newly designed routing method. Therefore, $A G N A$ can be readily applied to the setting of multiple-block warehouse. 


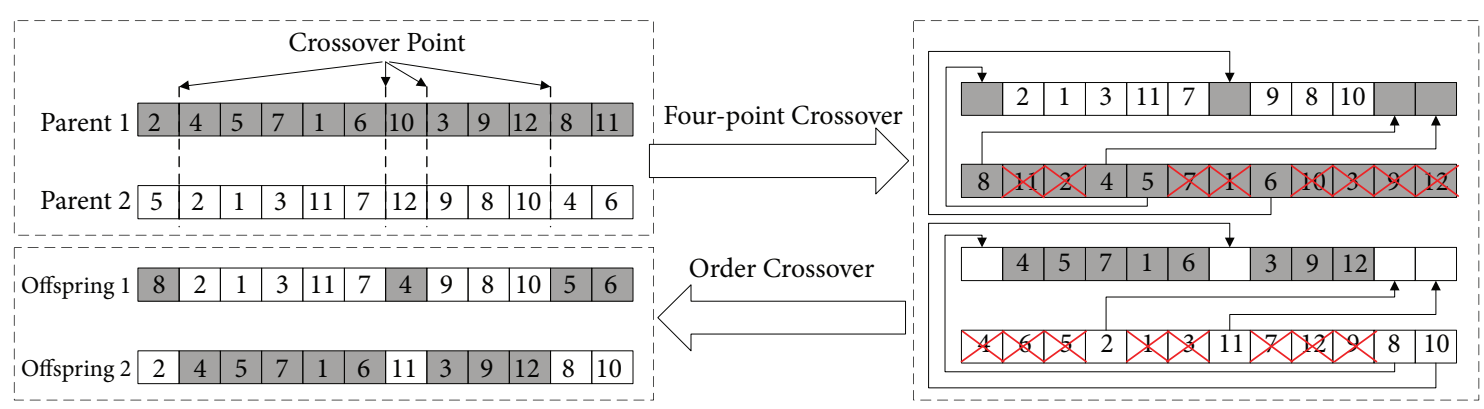

(a) Cross operation

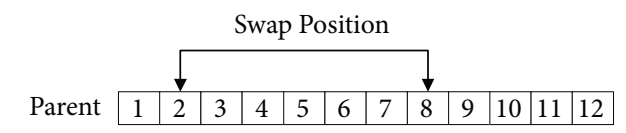

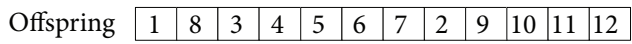

(b) Mutation operation

FIGURE 8: The illustration of crossover and mutation.

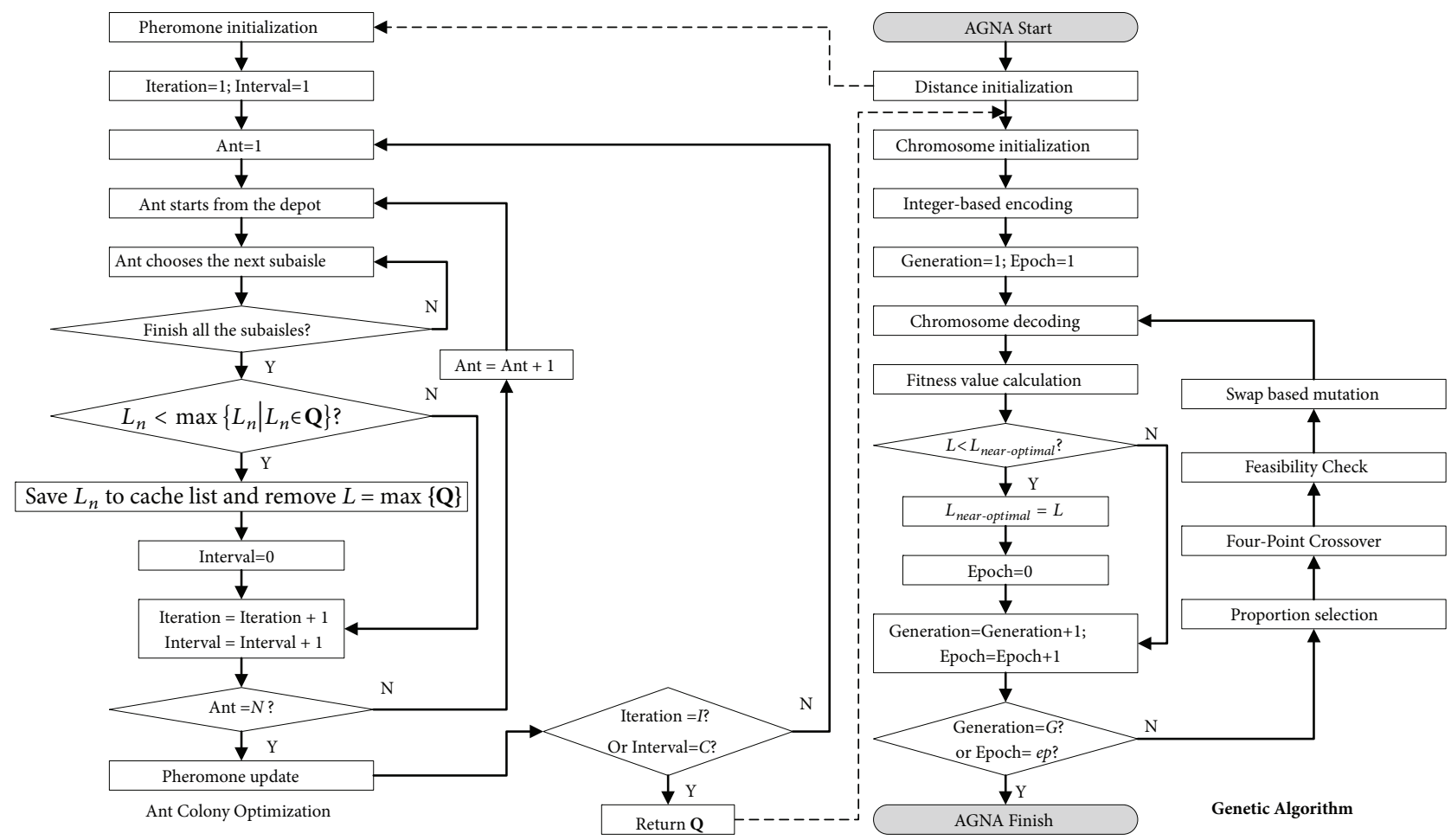

Figure 9: The flowchart of $A G N A$.

5.3. Discussions. Based on the results, we can analyze the effects of the following parameters.

(1) Access Restriction. In four scenarios, all the methods produce the same results. All of them are the layouts having only one Row, and access restriction is imposed on one side for all the blocks implying that all the subaisles are allowed to be visited from only one entrance. This means that only one identical access mode with the same travel distance will be chosen by all the routing methods. For the same reason, AGNA outperforms RNA and LNA in the other eight scenarios with fewer access restrictions (see Table 4). We calculate the percentage differences by $A / R=100 \times$ $(1-r(A) / r(R)), A / L=100 \times(1-r(A) / r(L))$, and $L / R=$ $100 \times(1-r(L) / r(R))$, where $r(R), r(L)$, and $r(A)$ refer to the average travel distances of $R N A, L N A$, and $A G N A$, respectively. The imposition of fewer access restrictions will increase the optimization space among these methods.

(2) Number of CNAs. Although the CNA is only responsible for the order pickers changing working aisles, AGNA produces better performance in a scenario with more CNAs. For 

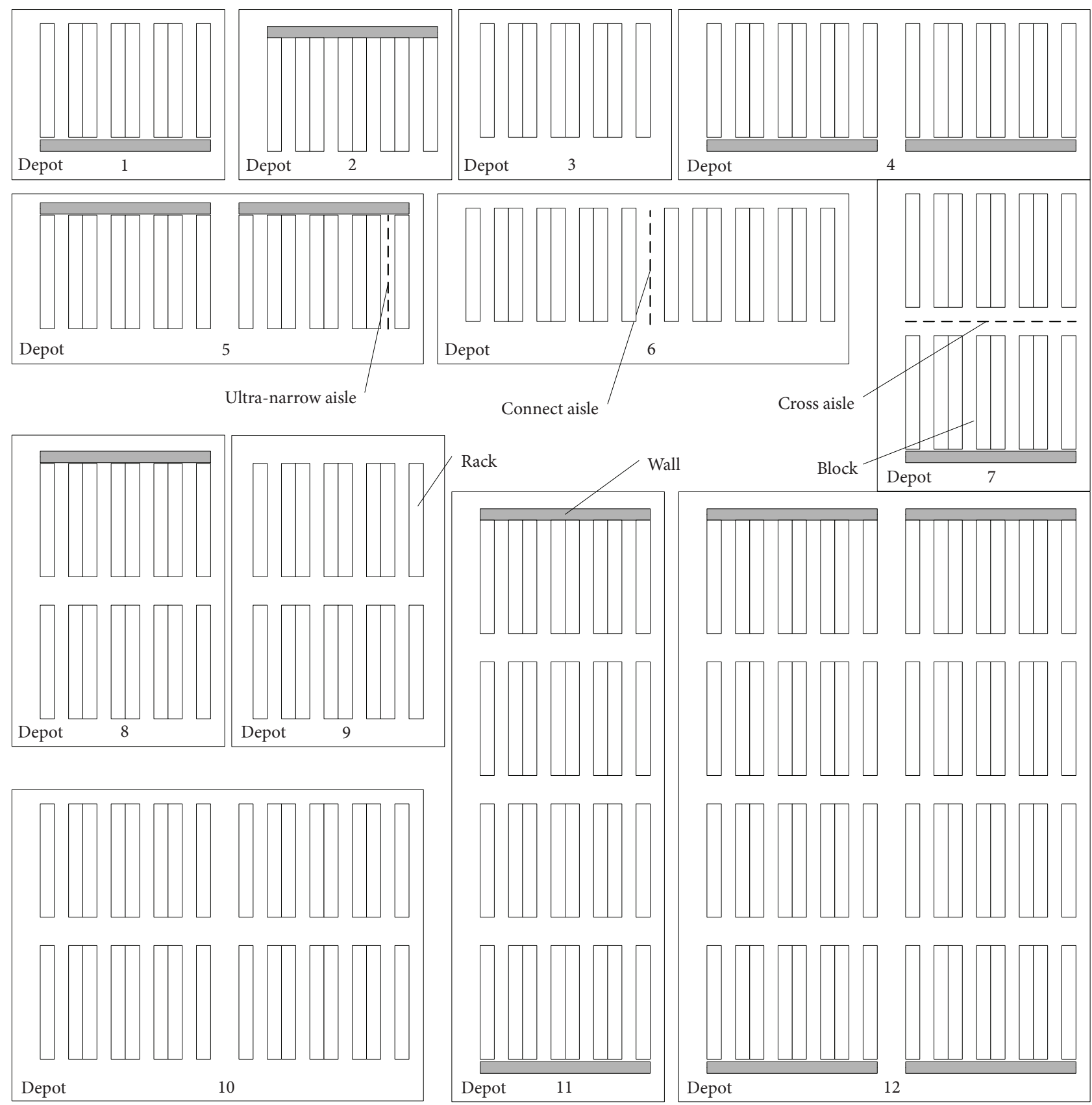

FIgURE 10: Warehouse scenarios concerned in the experiment.

instance, the mild increase in the number of CNAs from two (in the $3^{\text {rd }}$ scenario) to three (in the $6^{\text {th }}$ ) translates into a significant synchronous increase in the percentage difference between AGNA and RNA. Similar comparisons between $A G N A$ and RNA are shown in Figure 11. The implication of this observation is that the negative impacts of access restriction can be by mitigated by setting the optimal number of CNAs.

(3) Number of CAs. The presence of more CAs contributes to a reduced relative proportion of access restriction, by which the order pickers can enjoy greater flexibility. As shown in
Table 4, AGNA outperforms other methods in the scenarios with more CAs. If the access restriction is inevitable, we suggest that warehouse designers can set more CAs.

\section{Conclusions}

This paper studied a new kind of multiple-block warehouse with ultranarrow aisles as observed from one large-scale online retailer in China. In such a warehouse system, an order picker is prohibited from entering the ultranarrow aisles carrying a picking cart. In addition, we also take access restriction into account, which has been imposed 


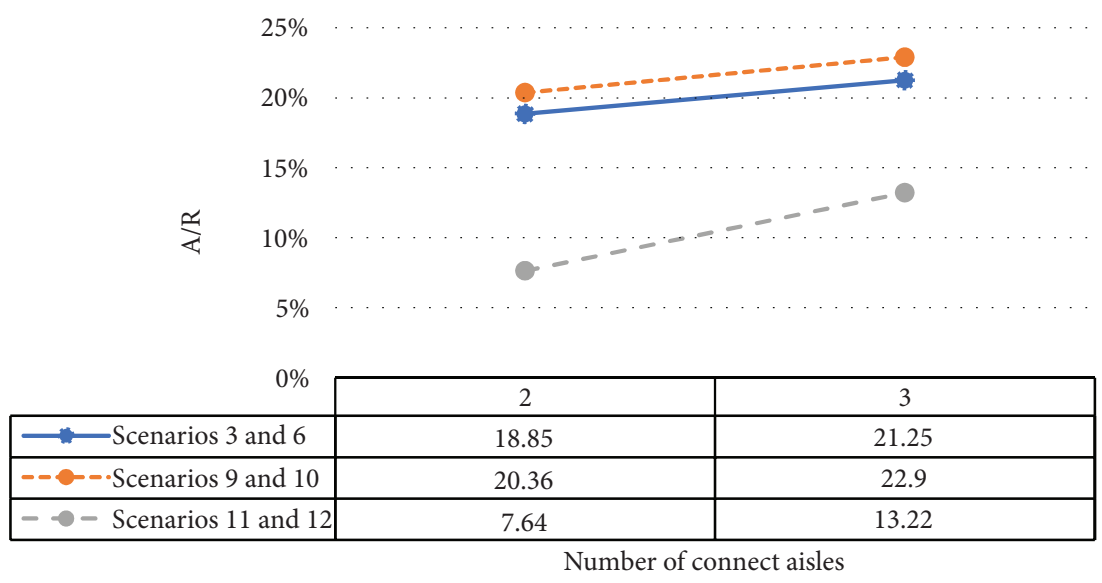

FIGURE 11: The percentage difference between $A G N A$ and $R N A$ in scenarios with two or three CNAs.

TABLE 1: Detailed configurations of warehouse scenarios.

\begin{tabular}{|c|c|c|c|c|c|}
\hline Layout scenario & Number of blocks & Number of cross aisles & Number of connect aisles & Front restriction & Back restriction \\
\hline 1 & 1 & 1 & 2 & Yes & No \\
\hline 2 & 1 & 1 & 2 & No & Yes \\
\hline 3 & 1 & 2 & 2 & No & No \\
\hline 4 & 2 & 1 & 3 & Yes & No \\
\hline 5 & 2 & 1 & 3 & No & Yes \\
\hline 6 & 2 & 2 & 3 & No & No \\
\hline 7 & 2 & 2 & 2 & Yes & No \\
\hline 8 & 2 & 2 & 2 & No & Yes \\
\hline 9 & 2 & 3 & 2 & No & No \\
\hline 10 & 4 & 3 & 3 & No & No \\
\hline 11 & 4 & 3 & 2 & Yes & Yes \\
\hline 12 & 8 & 3 & 3 & Yes & Yes \\
\hline
\end{tabular}

TABLE 2: The parameter setting for the AGNA algorithm.

\begin{tabular}{lcc}
\hline Parameter & $A C O$ & $G A$ \\
\hline Population & $|\boldsymbol{\Omega}|$ & $|\boldsymbol{\Omega}|$ \\
Iterations $(I)$ & $2000 *|\boldsymbol{\Omega}|$ & \\
Interval $(C)$ & $50 *|\boldsymbol{\Omega}|$ & \\
$\alpha$ & 1 & \\
$\beta$ & 5 & \\
$\rho$ & 0.15 & 0.75 \\
Crossover rate & & 0.1 \\
Mutation rate & & $100 *|\Omega|^{2}$ \\
$G$ & & $100 *|\Omega|$ \\
$e p$ & & \\
\hline
\end{tabular}

on some special pick aisles to limit the order pickers to visit by a designated entrance. In such a warehouse, the existing routing methods cannot be immediately applied without modification. This study designed a routing method by integrating $A C O$ and $G A$ to address those practical features with the aim to improve operational efficiency. Specifically, the core procedure of the AGNA lies in the identification of not only the best access mode but also the near-optimal visiting sequence and moving route that visits all the subaisles with picks.

A comprehensive simulation is deliberately designed to verify the efficacies of the proposed method. The input data for the simulation, including the warehouse configurations and order information, are collected from $D$ company. Moreover, two improved dedicated heuristics were also examined for comparison. By simulation, we found that AGNA can significantly improve the picking efficiency in most scenarios. Additionally, the mechanism of AGNA which deals with the access restriction can be deployed in the warehouses with traditional aisles as well. Moreover, it appears that minimizing access restrictions by setting more $C A$ s and $C N A s$ can effectively optimize the picking-service efficiency.

In this paper, the proposed method is developed for already-designed warehouse layouts. If the ultranarrow aisles and access restriction are inevitable, designing the optimal layout is a problem that is worthy of further research. Moreover, the designation of storage assignment and orderbatching methods in the setting of such a warehouse system also deserves ongoing further research.

\section{Data Availability}

The data will be accessible upon request. 
TABLE 3: The average length of travel distances for all the methods (unit: meter).

\begin{tabular}{lccr}
\hline Layout scenario & RNA & LNA & AGNA \\
\hline 1 & 247.65 & 247.65 & 247.65 \\
2 & 231.14 & 231.14 & 231.14 \\
3 & 208.90 & 208.90 & 171.55 \\
4 & 381.48 & 381.48 & 381.48 \\
5 & 370.02 & 370.02 & 370.02 \\
6 & 411.86 & 411.86 & 346.48 \\
7 & 296.15 & 296.15 & 263.05 \\
8 & 261.81 & 262.78 & 261.64 \\
9 & 313.63 & 312.04 & 263.48 \\
10 & 495.13 & 492.15 & 409.51 \\
11 & 341.70 & 340.67 & 329.56 \\
12 & 534.14 & 532.94 & 498.92 \\
\hline
\end{tabular}

TABLE 4: The percentage differences between different methods (unit: \%).

\begin{tabular}{lccc}
\hline Layout scenario & $A / R$ & $A / L$ & $L / R$ \\
\hline 1 & 0 & 0 & 0 \\
2 & 0 & 0 & 0 \\
3 & 17.88 & 17.88 & 0 \\
4 & 0 & 0 & 0 \\
5 & 0 & 0 & 0 \\
6 & 15.87 & 15.87 & 0 \\
7 & 11.18 & 11.18 & 0.43 \\
8 & 0.06 & 0.43 & -0.37 \\
9 & 15.99 & 15.56 & 0.51 \\
10 & 17.29 & 16.79 & 0.6 \\
11 & 3.55 & 3.26 & 0.3 \\
12 & 6.59 & 6.38 & 0.22 \\
\hline
\end{tabular}

\section{Conflicts of Interest}

The authors declare that they have no conflicts of interest.

\section{Acknowledgments}

This work was supported by the National Natural Science Foundation of China [Grants nos. 71701213 and 71401181] and the MOE (Ministry of Education in China) Project of Humanities and Social Sciences [Grants nos. 15YJC630008 and 14YJC630136].

\section{References}

[1] J. L. García, A. Alvarado, J. Blanco, E. Jiménez, A. A. Maldonado, and G. Cortés, "Multi-attribute evaluation and selection of sites for agricultural product warehouses based on an analytic hierarchy process," Computers and Electronics in Agriculture, vol. 100, pp. 60-69, 2014.

[2] R. De Santis, R. Montanari, G. Vignali, and E. Bottani, "An adapted ant colony optimization algorithm for the minimization of the travel distance of pickers in manual warehouses," European Journal of Operational Research, vol. 267, no. 1, pp. 120-137, 2018.
[3] J. A. Tompkins, J. A. White, Y. A. Bozer, and J. M. A. Tanchoco, Facilities Planning, John Wiley \& Sons, 2010.

[4] F. Chen, Y. Wei, and H. Wang, "A heuristic based batching and assigning method for online customer orders," Flexible Services and Manufacturing Journal, vol. 30, no. 4, pp. 640-685, 2018.

[5] J. Zhang, X. Wang, and K. Huang, "On-line scheduling of order picking and delivery with multiple zones and limited vehicle capacity," OMEGA - The International Journal of Management Science, vol. 79, pp. 104-115, 2018.

[6] C. G. Petersen II, "An evaluation of order picking routeing policies," International Journal of Operations and Production Management, vol. 17, no. 11, pp. 1098-1111, 1997.

[7] K. Gue, R. Meller, and J. Skufca, "The effects of pick density on order picking areas with narrow aisles," Institute of Industrial Engineers (IIE). IIE Transactions, vol. 38, no. 10, pp. 859-868, 2006.

[8] P. J. Parikh and R. D. Meller, "Estimating picker blocking in wide-aisle order picking systems," Institute of Industrial Engineers (IIE). IIE Transactions, vol. 41, no. 3, pp. 232-246, 2009.

[9] P. J. Parikh and R. D. Meller, "A travel-time model for a persononboard order picking system," European Journal of Operational Research, vol. 200, no. 2, pp. 385-394, 2010.

[10] S. Hong, A. L. Johnson, and B. A. Peters, "Batch picking in narrow-aisle order picking systems with consideration for 
picker blocking," European Journal of Operational Research, vol. 221, no. 3, pp. 557-570, 2012.

[11] F. Chen, H. Wang, C. Qi, and Y. Xie, "An ant colony optimization routing algorithm for two order pickers with congestion consideration," Computers \& Industrial Engineering, vol. 66, no. 1, pp. 77-85, 2013.

[12] F. Chen, H. Wang, Y. Xie, and C. Qi, "An ACO-based online routing method for multiple order pickers with congestion consideration in warehouse," Journal of Intelligent Manufacturing, vol. 27, no. 2, pp. 389-408, 2016.

[13] F. Chen, G. Xu, and Y. Wei, "Heuristic routing methods in multiple-block warehouses with ultra-narrow aisles and access restriction," International Journal of Production Research, vol. 57, no. 1, pp. 228-249, 2018.

[14] R. de Koster, T. Le-Duc, and K. J. Roodbergen, "Design and control of warehouse order picking: a literature review," European Journal of Operational Research, vol. 182, no. 2, pp. 481-501, 2007.

[15] R. W. Hall, "Distance approximations for routing manual pickers in a warehouse," Institute of Industrial Engineers (IIE). IIE Transactions, vol. 25, no. 4, pp. 76-87, 1993.

[16] N. Gademann and S. van de Velde, "Order batching to minimize total travel time in a parallel-aisle warehouse," Institute of Industrial Engineers (IIE). IIE Transactions, vol. 37, no. 1, pp. 6375, 2005.

[17] A. Alvarado-Iniesta, J. L. Garcia-Alcaraz, M. I. RodriguezBorbon, and A. Maldonado, "Optimization of the material flow in a manufacturing plant by use of artificial bee colony algorithm," Expert Systems with Applications, vol. 40, no. 12, pp. 4785-4790, 2013.

[18] H. Guo, C. Li, Y. Zhang, C. Zhang, and Y. Wang, "A nonlinear integer programming model for integrated location, inventory, and routing decisions in a closed-loop supply chain," Complexity, vol. 2018, Article ID 2726070, 17 pages, 2018.

[19] C. Huang, Y. Lan, Y. Liu et al., "A new dynamic path planning approach for unmanned aerial vehicles," Complexity, vol. 2018, Article ID 8420294, 17 pages, 2018.

[20] O. Kulak, Y. Sahin, and M. E. Taner, "Joint order batching and picker routing in single and multiple-cross-aisle warehouses using cluster-based tabu search algorithms," Flexible Services and Manufacturing Journal, vol. 24, no. 1, pp. 52-80, 2012.

[21] M. Matusiak, R. de Koster, L. Kroon, and J. Saarinen, "A fast simulated annealing method for batching precedenceconstrained customer orders in a warehouse," European Journal of Operational Research, vol. 236, no. 3, pp. 968-977, 2014.

[22] C. A. Valle, J. E. Beasley, and A. S. da Cunha, "Optimally solving the joint order batching and picker routing problem," European Journal of Operational Research, vol. 262, no. 3, pp. 817-834, 2017.

[23] C.-C. Lin, J.-R. Kang, C.-C. Hou, and C.-Y. Cheng, "Joint order batching and picker Manhattan routing problem," Computers \& Industrial Engineering, vol. 95, pp. 164-174, 2016.

[24] E. H. Grosse, C. H. Glock, and R. Ballester-Ripoll, "A simulated annealing approach for the joint order batching and order picker routing problem with weight restrictions," International Journal of Operations and Quantitative Management, vol. 20, no. 2, pp. 65-83, 2014.

[25] T.-L. Chen, C.-Y. Cheng, Y.-Y. Chen, and L.-K. Chan, "An efficient hybrid algorithm for integrated order batching, sequencing and routing problem," International Journal of Production Economics, vol. 159, pp. 158-167, 2015.
[26] C.-Y. Cheng, Y.-Y. Chen, T.-L. Chen, and J. Jung-Woon Yoo, "Using a hybrid approach based on the particle swarm optimization and ant colony optimization to solve a joint order batching and picker routing problem," International Journal of Production Economics, vol. 170, pp. 805-814, 2015.

[27] J. Li, R. Huang, and J. B. Dai, "Joint optimisation of order batching and picker routing in the online retailer's warehouse in China," International Journal of Production Research, vol. 55, no. 2, pp. 447-461, 2017.

[28] A. Scholz, S. Henn, M. Stuhlmann, and G. Wäscher, "A new mathematical programming formulation for the single-picker routing problem," European Journal of Operational Research, vol. 253, no. 1, pp. 68-84, 2016.

[29] T.-H. Chang, H.-P. Fu, and K.-Y. Hu, "A two-sided picking model of M-AS/RS with an aisle-assignment algorithm," International Journal of Production Research, vol. 45, no. 17, pp. 39713990, 2007.

[30] X. Xu, T. Liu, K. Li, and W. Dong, "Evaluating order throughput time with variable time window batching," International Journal of Production Research, vol. 52, no. 8, pp. 2232-2242, 2014.

[31] S. Henn and V. Schmid, "Metaheuristics for order batching and sequencing in manual order picking systems," Computers \& Industrial Engineering, vol. 66, no. 2, pp. 338-351, 2013.

[32] K. J. Roodbergen and R. De Koster, "Routing methods for warehouses with multiple cross aisles," International Journal of Production Research, vol. 39, no. 9, pp. 1865-1883, 2001.

[33] J. C.-H. Pan, P.-H. Shih, and M.-H. Wu, "Order batching in a pick-and-pass warehousing system with group genetic algorithm," OMEGA - The International Journal of Management Science, vol. 57, pp. 238-248, 2015.

[34] K. J. Roodbergen and R. de Koster, "Routing order pickers in a warehouse with a middle aisle," European Journal of Operational Research, vol. 133, no. 1, pp. 32-43, 2001. 


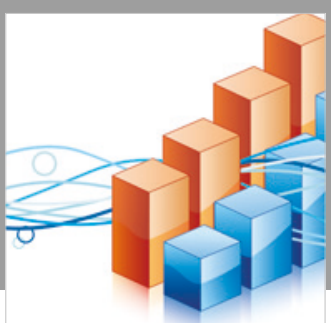

Advances in

Operations Research

\section{-n-m}
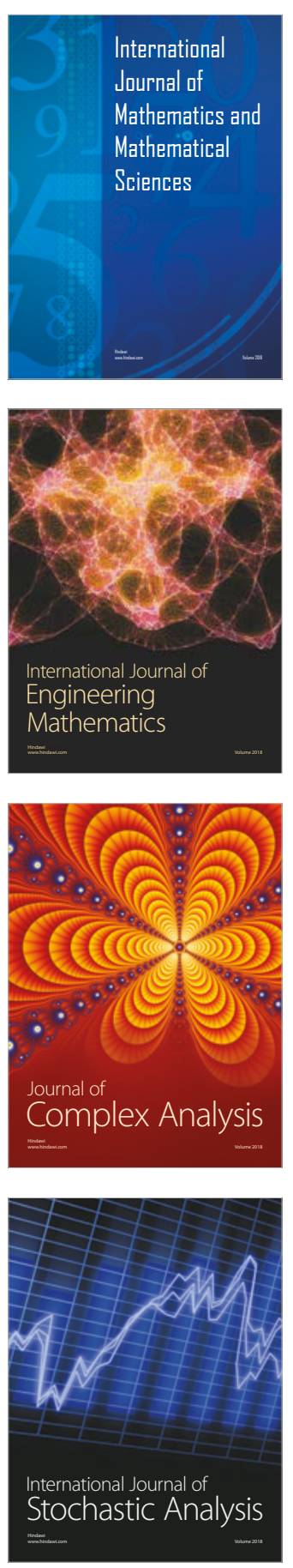
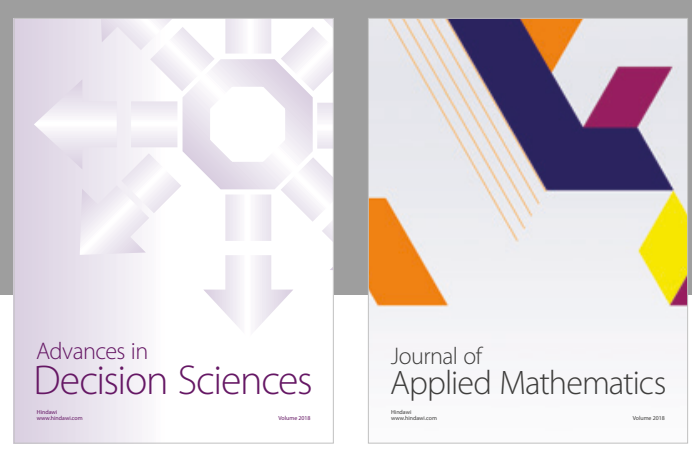

Journal of

Applied Mathematics
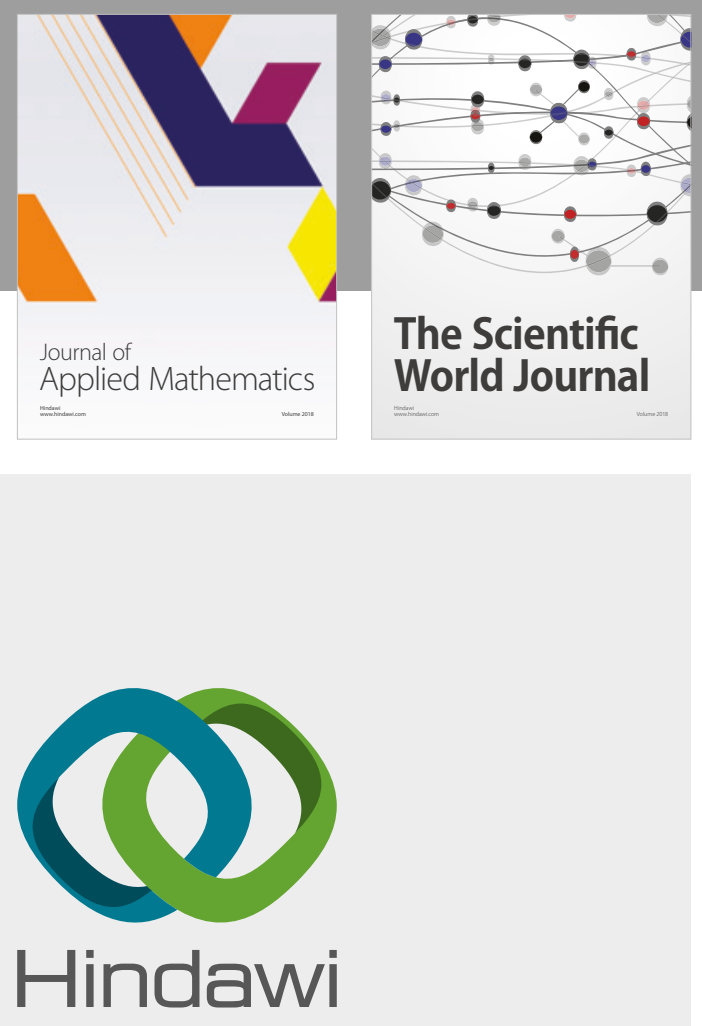

Submit your manuscripts at

www.hindawi.com

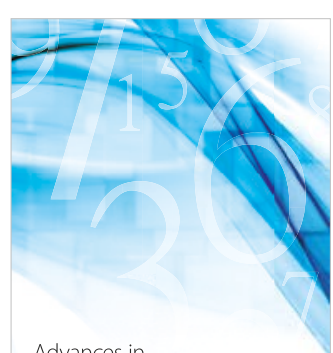

Advances in
Numerical Analysis
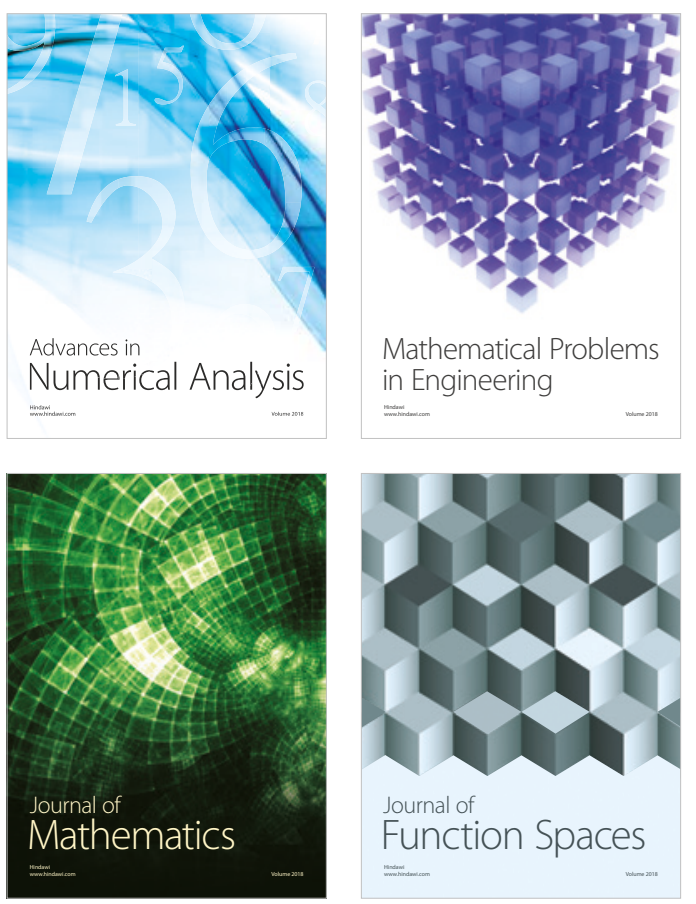

Mathematical Problems in Engineering

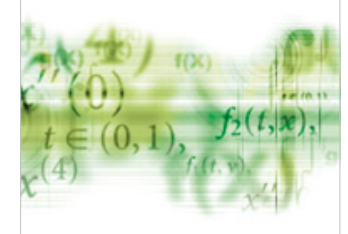

International Journal of

Differential Equations

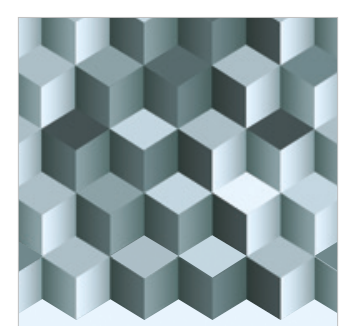

Journal of

Function Spaces
The Scientific

World Journal

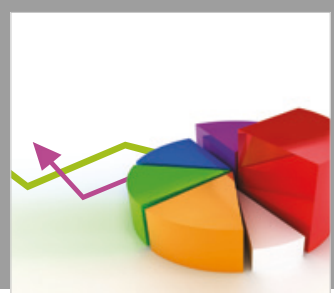

Journal of

Probability and Statistics
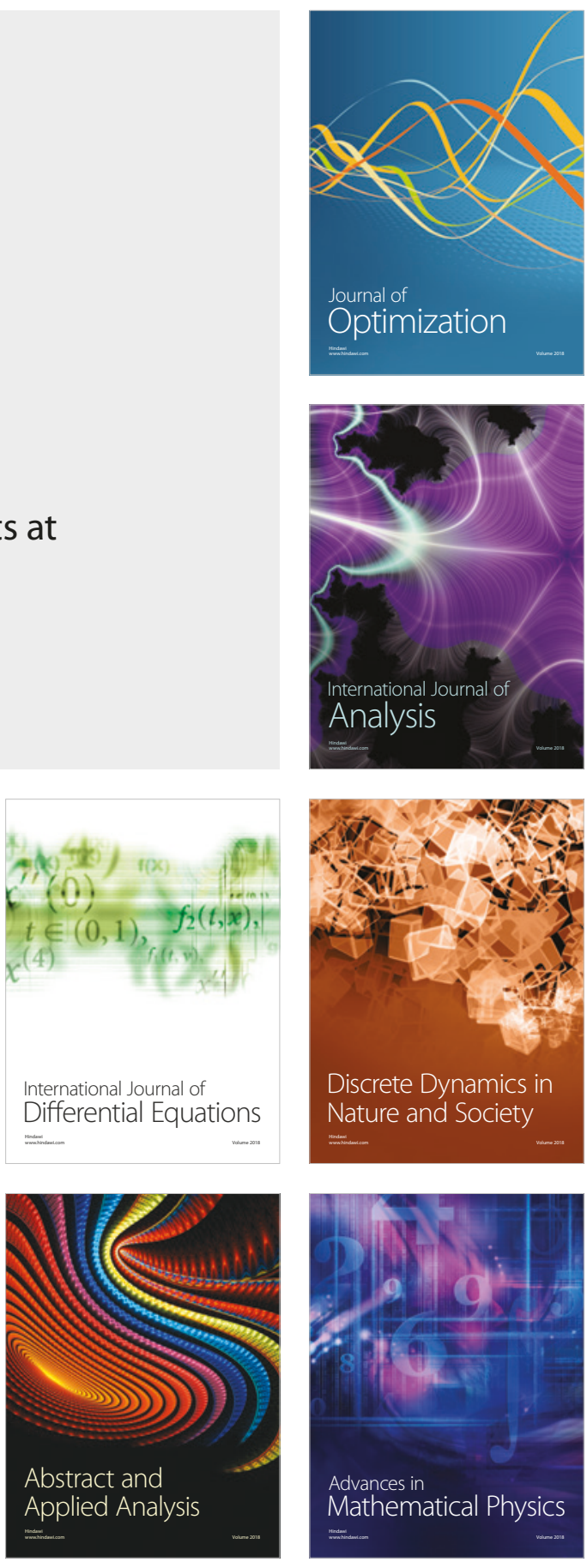